\title{
The effects of strong irrelevant thirst on food-rewarded instrumental performance
}

\author{
ELIZABETH D. CAPALDI, JOHN R. HOVANCIK, and ENGIN O. LAMB \\ Purdue University. West Lafarette. Indiana 47907
}

\begin{abstract}
In Experiment I, rats received one food rewarded trial per day in a runway. One group received all its trials under hunger (Group $H$ ); the second group received a random half of its trials under hunger and the other half of its trials under hunger plus thirst (Group H-HT). Group H-HT ultimately ran slower on HT trials than on $H$ trials. In Experiment II, the effects of shifting from $H$ to $H T$-and vice versa were examined in a five-phase design. In general, rats run under $H$ ran faster than rats run under $\mathrm{HT}$, and shifts from $\mathrm{H}$ to $\mathrm{HT}$ produced rapid decreases in speed, while shifts from $\mathrm{HT}$ to $\mathrm{H}$ produced extremely slow increases in speed. The results of both experiments were interpreted as indicating that the reward value of food is greater under $\mathrm{H}$ than under HT and that the manipulation $\mathrm{H}$ vs. HT may be viewed as theoretically similar to manipulation of reward magnitude.
\end{abstract}

It has been shown that rats deprived of both food and water (hunger plus thirst, HT) eat less when food alone is given and are inferior in food-rewarded instrumental performance to rats deprived only of food (hunger, $\mathrm{H}$ ) (e.g., Bolles \& Morlock, 1960; Kendler \& Law, 1950; Levine, 1956; Verplanck \& Hayes, 1953), at least when thirst is strong ( $22 \mathrm{~h}$ deprivation or more). These results may be interpreted as indicating that the reward value of food is less under HT than under $H$. The purpose of the two experiments reported here was to determine if the effects on performance of manipulating $\mathrm{H}$ vs. HT are similar to the effects of manipulating reward magnitude. If the effects on performance of these two manipulations are similar, it would then seem feasible to ask whether or not these similar effects are produced through the same mechanisms.

Although the results of previous studies investigating instrumental performance as a function of $\mathrm{H}$ vs. HT are compatible with the interpretation that the reward value of food is less under HT than under $\mathrm{H}$, they are also compatible with other interpretations for two reasons. First, previous studies measuring instrumental performance as a function of $\mathrm{H}$ vs. HT have employed more than one trial a day. Accordingly, in previous studies, it is possible that $\mathrm{HT}$ rats were inferior to $\mathrm{H}$ rats because of an aftereffect of having consumed food on Trial 1, an aftereffect (e.g., dry mouth) which limited their subsequent food consumption and thus produced slow running. This hypothesis is supported by Bolles and Morlock's (1960) finding that animals run to food under $\mathrm{H}$ on some days and under $\mathrm{H}$ plus strong $\mathrm{T}$ on other days ran equally rapidly under $\mathrm{H}$ and $\mathrm{HT}$ on Trial 1 of the day, but ran more slowly under HT than under $\mathrm{H}$ on

This research was supported in part by Grant MH 23446-01 to the first author from the National Institute of Mental Health. Experiment II is based in part on a thesis by the thind author under the direction of the first author submitted to the graduate school of Pundue University in partial fulfillment of the requirement for the master's degree. Reprints may be obtained from Elizabeth D. Capaldi, Department of Psychological Sciences, Purdue University, West Lafayette, Indiana 47907. later trials on the day. If this interpretation of previous results is correct. we would expect that the usual inferior performance under $\mathrm{HT}$ relative to $\mathrm{H}$ when more than one trial a day is given would not occur at one trial a day. If, however, the reward value of food is lower under HT than under $\mathrm{H}$, performance under $\mathrm{HT}$ should be inferior to that under $H$ even at one trial a day. Experiment $I$ is a test of this proposition.

A second limitation of previous studies comparing instrumental performance under $\mathrm{H}$ and $\mathrm{H}$ plus strong thirst is that all studies reporting acquisition data have employed a constant amount of time in the baited goalbox as a reward (20 sec in Bolles \& Morlock, 1960; Kendler, 1947; Kendler \& Law, 1950; and Levine, 1956). If HT animals eat more slowly than $\mathrm{H}$ animals, HT animals would consume less than $H$ animals in a fixed period of time and would thus receive a smaller magnitude of reward on all trials. Thus, the inferiority of HT to $\mathrm{H}$ animals in previous studies could reflect their receiving a smaller magnitude of reward. For this reason, Experiment I also equated for amount of food consumed on $\mathrm{H}$ and HT trials.

\section{EXPERIMENT I}

\section{Method}

Subjects. The subjects were 26 naive male albino rats approximately 90 days old upon arrival from the Holtzman Company, Madison, Wisconsin.

Apparatus. The apparatus was a runway painted a flat gray throughout, $190.5 \times 10.2 \mathrm{~cm}$ with $22.9-\mathrm{cm}$ high sides, covered with 1.3-cm hardware cloth. A $20.3-\mathrm{cm}-$ long floor treadle, suspended over a microswitch, constituted the initial portion of the alley. When the treadle was depressed by the weight of the rat, a $.01-\mathrm{sec}$ clock was started. This clock was stopped (start time) and another started when the rat interrupted a photobeam $13.0 \mathrm{~cm}$ beyond the treadle. The second clock was stopped (run time) and a third one started when the rat interrupted a second photobeam located $106.7 \mathrm{~cm}$ from the first. Interruption of the third photobeam stopped the third clock (goal time). The third photobeam was $38.7 \mathrm{~cm}$ beyond the second photobeam and $7.5 \mathrm{~cm}$ in front of a gray block of wood with a hole, $3.17 \mathrm{~cm}$ in 
diam and $1.9 \mathrm{~cm}$ deep, which served as a foodcup. A manually lowered guillotine door, $36.8 \mathrm{~cm}$ from the rear of the goal compartment, served to confine the rat to the goal area. The times from the three clocks were summed to obtain total time.

Procedure. Upon arrival at the laboratory, subjects were caged individually and given ad-lib access to food and water for a period of 9 days. On the 9 th day, subjects were weighed and assigned to two groups $(\mathrm{N}=13)$ matched on the basis of ad-lib weight. All subjects were placed on a deprivation schedule consisting of $12 \mathrm{~g}$ of Wayne Lab Blox and $2 \mathrm{~h}$ of ad-lib access to water daily. Subjects were maintained on this schedule for 14 days preceding the start of acquisition. On the 3 days immediately prior to acquisition, subjects were handled individually for $90 \mathrm{sec}$ each. In acquisition, each subject received one trial reinforced with $22.045-\mathrm{g}$ Noyes pellets each day for a total of 80 days. Animals were removed from the goalbox immediately after all the pellets were consumed. Consummatory times, measured from the time the subject entered the goalbox until the reward was completely consumed, were recorded on all training trials. Half of the trials for Group H-HT and all of the trials for Group $\mathrm{H}$ were administered following $30 \mathrm{~min}$ free access to water $(\mathrm{H})$. The remaining half of the trials for Group H-HT were administered preceding the 2 -h water access period (HT). The schedule of H and HT trials for Group H-HT was random with the restriction that the greatest number of consecutive trials under either deprivation condition was three. Running order was randomized within squads of three and four subjects. Each squad was run at the same time each day. Following each trial, subjects were returned to their home cages and allowed access to water for the remaining portion of the 2-h water access period. The daily ration minus the amount consumed in the apparatus was fed to subjects $15 \mathrm{~min}$ after completion of training.

\section{Results}

All times were converted to speeds $(1 / \mathrm{sec})$, and speeds in each alley section were analyzed in a between-within analysis of variance including groups as the between factor and $\mathrm{H}$ vs. HT trials and trial blocks as within factors. As Group $\mathrm{H}$ received all its trials under $\mathrm{H}$, for purposes of analysis, trials on which Group H-HT ran under HT were designated as HT trials for Group $\mathrm{H}$. Results in all alley sections were substantially the same, although differences were smaller in the goal section, thus only total speeds are presented here. Figure 1 shows the mean total speed for each group in blocks of eight $\mathrm{H}$ trials and eight HT trials for Group H-HT and in blocks of 16 trials for Group H. As can be seen in Figure 1, Group H-HT ultimately ran slower on HT trials than on $\mathrm{H}$ trials. At the end of training, Group H-HT's performance on HT trials was below that of Group $\mathrm{H}$, while Group H-HT's performance on $\mathrm{H}$ trials was approximately equal to that of Group $H$.

Analysis of the last three blocks of trials indicated that the groups did not differ significantly, $F<1$. However, the difference due to drive ( $\mathrm{H}$ vs. HT trials) was significant, $F(1,24)=26.95, p<.001$, as was the interaction of drive and groups, $F(1,24)=52.96$, $\mathrm{p}<.001$. This interaction was due to Group H-HT running significantly slower on $\mathrm{HT}$ trials than on $\mathrm{H}$ trials, $F(1,12)=80.25, \mathrm{p}<001$, while Group $\mathrm{H}$ ran nondifferentially on ordinally comparable trials,

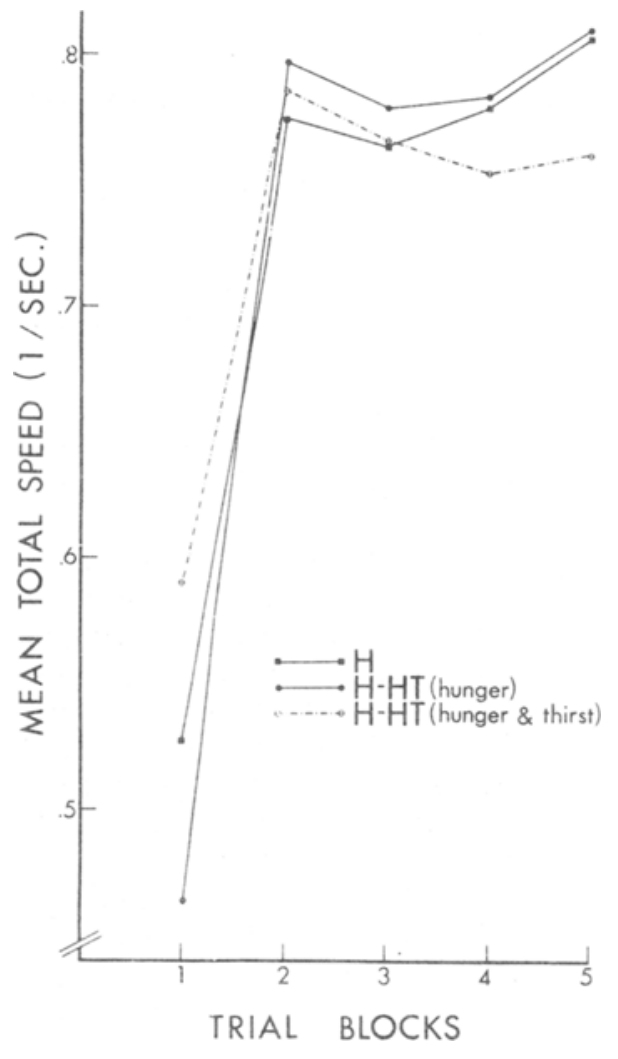

Figure 1. Mean total speed for Group H-HT on hunger and hunger plus thirst trials (eight trials per block) and for Group $\mathrm{H}$ on hunger trials (sixteen trials per block).

$F(1,12)=2.11, p>.10$. Subsequent Newman-Keuls tests $(\mathrm{p}<.01)$ indicated that Group H-HT ran significantly slower on HT trials than did Group $H$, while Group H-HT did not differ significantly from Group $\mathrm{H}$ on $\mathrm{H}$ trials.

As can also be seen in Figure 1, early in acquisition, Group H-HT ran faster on HT trials than on $H$ trials. Although not shown in Figure 1, Group $\mathrm{H}$ also ran faster on trials comparable to Group H-HT's HT trials than on trials comparable to Group H-HT's H trials early in acquisition. This difference for both groups is most likely due to the fact that the first three trials, when speeds were the slowest, were $\mathrm{H}$ trials. On the first trial block, the difference due to drive ( $\mathrm{H}$ vs. HT trials) was significant $F(1,24)=4.36, p<.001$, both groups running significantly faster on HT trials than on $\mathrm{H}$ trials-Group $\mathrm{H}: \mathrm{F}(1,12)=419.32, \mathrm{p}<.001$, Group H-HT: $\quad F(1,12)=132.46, \quad p<.001$. This difference was equally large for both groups, Groups by Drive, $F(1,24)=2.92, p>.10$. By the second trial block, Group H-HT began to run more slowly on HT trials than on $\mathrm{H}$ trials, while Group $\mathrm{H}$ continued to run slightly faster on trials comparable to Group H-HT's HT trials than on trials comparable to Group H-HT's H trials, producing a significant Groups by Drive 
interaction, $F(1,24)=6.72, p<.02$ on the second trial block. Neither the difference due to drive nor the difference due to groups was significant on the second trial block, Fs $<1$.

Analysis of consummatory times over all five trial blocks indicated that Group H-HT took significantly longer to eat on $\mathrm{HT}$ trials than on $\mathrm{H}$ trials, $\mathrm{F}(1,12)=30.31, \mathrm{p}<.001$, while Group $\mathrm{H}$ took significantly longer to eat on trials comparable to Group H-HT's $H$ trials than on trials comparable to Group H-HT's HT trials, $F(1,12)=37.86, p<.001$. These differences resulted in a significant Groups by Drive interaction, $F(1,24)=64.36, p<.001$. The difference for Group $\mathrm{H}$ was entirely due to the early trial blocks. Consummatory time decreased with trials, and as the first three trials were $\mathrm{H}$ trials, consummatory times were longer on $\mathrm{H}$ than on HT trials for both groups on the first trial block, this difference being significantly larger for Group $H$ than for Group H-HT, $F(1,24)=12.52, p<.01$. On the later trial blocks, Group $\mathrm{H}$ did not differ on the two types of trials, while Group H-HT took significantly longer to eat on HT than on $H$ trials, smallest $F(1,12)=12.89, p<.01$, on Trial Block 5. The fact that consummatory times were longer on HT trials than on $\mathrm{H}$ trials suggests that it is inadvisable to employ a constant amount of time in the baited goalbox as a reward when $\mathrm{H}$ and HT conditions are compared.

\section{Discussion}

The results of Experiment I suggest that dry food is less reinforcing when experienced under HT than when experienced under $\mathrm{H}$ and that rats can anticipate this differential reinforcing effect of food. This result, unlike previous results, cannot be attributed to an unpleasant aftertaste of food under HT, because the intertrial interval was $24 \mathrm{~h}$; nor can it be attributed to different amounts of food being consumed on $\mathrm{H}$ and HT trials, as 22 pellets were consumed on all trials. Thus, the results of Experiment I support the hypothesis that the $\mathrm{H}$ vs. HT manipulation affects reward value in a manner similar to other manipulations of reward value, e.g., reward magnitude.

The results of Experiment I do not appear attributable to hunger drive being lower on HT trials than on $\mathrm{H}$ trials or to the drive stimulus characteristic of thirst eliciting a competing response. If either of these hypotheses were correct, it would be expected that speeds on HT trials would have been inferior to those on $\mathrm{H}$ trials early in training. However, early in training, performance on HT trials was superior to that on $\mathrm{H}$ trials. Although this effect was presumably due to acquisition of the running response, it seems unlikely that if acquisition effects were not present, speeds would have been faster on $\mathrm{H}$ trials than on $\mathrm{HT}$ trials for Group H-HT. If there had been a tendency for speeds to be faster on $\mathrm{H}$ than on HT trials for Group H-HT, it would be expected that the difference early in training favoring speeds on HT trials would have been smaller for Group H-HT (the acquisition effect being opposed by the presumed faster speeds on $\mathrm{H}$ trials) than for Group $\mathrm{H}$ (acquisition effect alone). Yet, the superiority of speeds on HT trials to those on $\mathrm{H}$ trials early in training was equally large for both groups. In addition, the same amount of food (12 g) was consumed each day and body weight did not vary, thus it seems unlikely that the hunger drive varied. And although the fact that consummatory times were longer on HT trials than on $\mathrm{H}$ trials is consistent with the interpretation that the hunger drive was lower on HT trials than on $\mathrm{H}$ trials, this is also consistent with the hypothesis that dry food is less palatable and thus less reinforcing under HT than under $\mathrm{H}$. For these reasons, this interpretation favored here is that dry food is less reinforcing when experienced under HT than when experienced under $\mathrm{H}$ alone and that what is being learned in the present situation is an inhibitory reaction conditioned to the stimuli associated with HT. This experimental situation may thus be viewed as analogous to differential conditioning in which large reward is associated with one cue (e.g., black alley), and small reward with a different cue (e.g., white alley). Here, a large reward value was associated with the cues characteristic of $\mathrm{H}$ while a small reward value was associated with the cues characteristic of HT.

\section{EXPERIMENT II}

If manipulation of reward value by means of drive manipulations is functionally identical to manipulating reward value by changing reward magnitude, shifts from $\mathrm{H}$ to $\mathrm{HT}$ and vice versa should produce effects similar to shifts from large to small reward and vice versa. The effects of shifts in reward magnitude have received much study, and these effects have been of critical importance in developing theories of reward magnitude (e.g., Capaldi \& Lynch, 1967). Thus, to evaluate the similarity between the effects of $\mathrm{H}$ vs. HT and reward magnitude on performance, it would seem of importance to determine the effects of shifts from $H$ to $H T$ and vice versa. Repeated shifts from $H$ to $H T$ and vice versa have not been investigated previously.

The following effects are obtained with shifts in reward magnitude (e.g., Capaldi \& Lynch, 1967) and were looked for in Experiment II with shifts from $\mathrm{H}$ to HT and vice versa: (1) if animals are shifted from large to small reward, their performance drops temporarily below the level of a small reward control group's performance (negative contrast effect); (2) preceding a block of large reward trials by a block of small reward trials reduces the size of the negative contrast effect (transfer downshift effect); (3) animals shifted from small to large reward increase in speed rapidly, usually to 
the level of a large reward control group (no positive contrast effect); and (4) preceding a block of small reward trials by a block of large reward trials increases the rate of the upshift in speed when reward is shifted from small to large (transfer upshift effect).

\section{Method}

Subjects. The subjects were 40 naive male albino rats, about 90 days old upon arrival from the Holtzman Company, Madison, Wisconsin.

Apparatus. The apparatus was the same as that employed in Experiment I.

Procedure. The deprivation schedule began on the 5th day (Day 1) following the subjects' arrival in the laboratory. Each day, each rat received $2 \mathrm{~h}$ free access to water. One-half hour after access to water began, the daily ration $(12 \mathrm{~g})$ was fed. On Days 15-17, each rat was handled individually for $90 \mathrm{sec}$. Immediately following handling, the 2-h access to water began; $15 \mathrm{~min}$ following access to water, each rat was fed $15.045 \mathrm{-g}$ pellets. After the pellets were consumed, the daily ration was fed. The amount of food consumed each day in pretraining was subtracted from the daily ration. On Day 18 , each rat received one trial rewarded with 15 pellets. On Day 19, experimental training began. Animals were weighed every day throughout the study.

There were five phases of experimental training, 9 days in Phase 1, 10 days in each of Phases 2 and 3, and 8 days in each of Phases 4 and 5. Each rat received three trials per day in each phase. The four groups are designated by the deprivation condition they experienced in each phase, either hunger, $\mathrm{H}$, or hunger plus thirst, HT. On $\mathrm{H}$ days, $30 \mathrm{~min}$ free access to water preceded the daily trials and $1 \frac{1 / 2}{h}$ of free access to water followed the daily trials. On HT days, the $2 \mathrm{~h}$ free access to water followed the daily trials. Rats were run in squads of four, composed of one animal from each group in Phases 1, 2, and 3, and composed of two animals from each group in Phases 4 and 5 , producing an intertrial interval of 4-6 min. On all days, rats in a given squad were fed their daily ration minus the amount consumed in the apparatus $15 \mathrm{~min}$ after their trials were completed. Rats were removed from the goalbox as soon as all the pellets were consumed. On Day 1 of Phase 1, each trial was rewarded with 15 pellets. Every trial thereafter was rewarded with 22 pellets.

Phases 1 and 2 comprised a 2 by 2 factorial combining $\mathrm{H}$ and HT in Phase 1 with $\mathrm{H}$ and HT in Phase 2. In Phase 3, the shifted groups were returned to their Phase 1 drive. In Phases 4 and 5, only two groups continued: Group H-H-H was shifted to HT in Phase 4, and Group HT-HT-HT continued under HT in Phase 4. In Phase 5, both of these groups were shifted to $\mathrm{H}$.

\section{Results}

All times were converted to speeds $(1 / \mathrm{sec})$. Results in all alley sections were essentially the same; thus, only total speeds are reported here. All analyses included trials within each day as a factor; however, since trials did not interact with any other variable, trials within a day are not mentioned further. Speeds in Phase 1 and Phase 2 were analyzed in a 2 (drive in Phase $1, \mathrm{H}$ vs. HT) by 2 (drive in Phase 2, $\mathrm{H}$ vs. HT) factorial between-within analysis of variance. Figure 2 shows the mean speed under $\mathrm{H}$ and HT in Phase 1 and the mean speed for each of the four groups in Phase 2. As can be seen in Figure 2, rats under $\mathrm{H}$ in Phase 1 ran faster than rats under $H T, F(1,36)=5.04, p<.03$, this difference

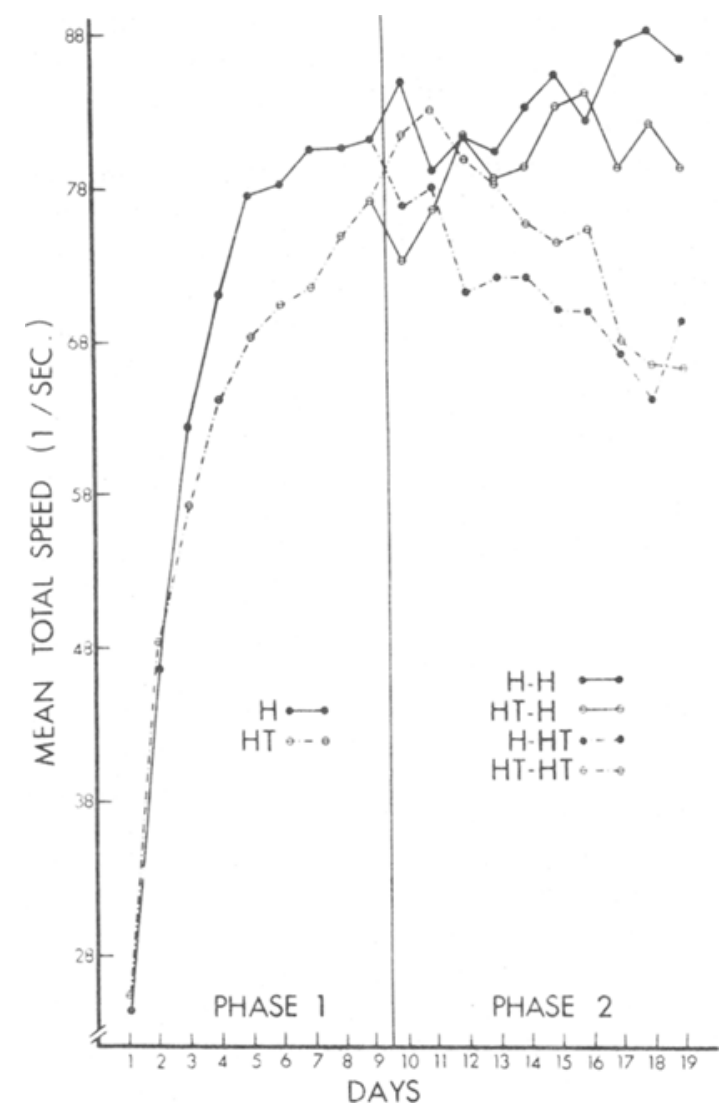

Figure 2. Mean total speed under the two drive conditions on each day in Phase 1 and for each of the four groups on each day of Phase 2.

growing larger over days, $F(8,288)=2.60, p<.01$. In Phase 1 , neither the difference due to Phase 2 drive (not yet experienced) nor the interaction between Phase 1 and Phase 2 drive were significant, $F<1$, $F(1,36)=3.32, p>.05$, respectively. In Phase 2 , as can be seen in Figure 2, rats shifted from $\mathrm{H}$ to HT dropped in speed below the level of Group HT-HT, while rats shifted from HT to $\mathrm{H}$, following an initial drop in speed, increased in speed to the level of Group $\mathrm{H}-\mathrm{H}$. Rats maintained under $\mathrm{H}$ continued to run faster than rats maintained under HT, although this difference decreased temporarily at the beginning of Phase 2. The last day of Phase 1 and the first day of Phase 2 were employed as the within factor in a between-within analysis of variance including drive in Phase 1 and drive in Phase 2 as between factors. The Days by Phase 1 Drive by Phase 2 Drive interaction was not significant in this analysis, $F<1$, indicating that the drop in speed for groups which experienced a change in drive was not significantly different from the increase in speed for the unshifted groups. Over all of Phase 2, because of the changing speeds of the groups for which drive was changed, the difference due to Phase 1 drive decreased 
over days, $F(9,324)=2.40, p<.02$, and the difference due to Phase 1 drive was not significant over all of Phase $2, F<1$. The difference due to Phase 2 drive was significant over all of Phase $2 F(1,36)=70.92$, $\mathrm{p}<.0001, \mathrm{H}$ in Phase 2 producing faster running than HT in Phase 2, this difference increasing over days, $F(9,324)=12.01, p<.001$. The interaction between Phase 1 drive and Phase 2 drive was also significant over all of Phase $2, F(1,36)=14.37, p<.001$. Newman-Keuls tests $(p<.05)$ indicated that the difference between each pair of groups was significant over all of Phase 2. On the last day of Phase 2, the difference between each pair of groups except Group H-HT vs. Group HT-HT was significant (Newman-Keuls, $p<.05$ ).

Figure 3 shows the mean speeds for each of the four groups on the last day of Phase 2 and on each day of Phase 3. As can be seen in Figure 3, Group H-H-H continued to run faster than Group HT-HT-HT throughout Phase 3. Group H-HT-H increased in speed very slowly throughout Phase 3, while Group HT-H-HT dropped immediately to the level of Group HT-HT-HT. Employing the last day of Phase 2 and the first day of Phase 3 as the within factor in a between-within analysis of variance including Phase 2 drive and Phase 3 drive as between factors, the Days by Phase 2 Drive by Phase 3 Drive interaction was significant, $F(1,36)=4.16$, $\mathrm{p}<.05$. Subsequent Newman-Keuls tests indicated that the significant interaction was due to Group HT-H-HT dropping significantly in speed from the last day of Phase 2 to the first day of Phase $3(p<.01)$, while no other group changed in speed significantly. Speeds in Phase 3 were analyzed in a 2 by 2 factorial between-within analysis of variance including Phase 3 drive ( $\mathrm{H}$ vs. $\mathrm{HT}$ ) and Phase 2 drive (H vs. $\mathrm{HT}$ ) as factors (Phase 3 drive was identical to Phase 1 drive for all groups). Over all of Phase 3 , the difference due to Phase 2 drive was significant, $\mathrm{H}$ in Phase 2 producing faster Phase 3 running than HT in Phase 2, $F(1,36)=61.99, p<.001$, this difference decreasing over days, $F(9,324)=3.44, p<.001$. The difference due to Phase 3 drive was also significant, $H$ producing faster running than $H T, F(1,36)=64.96, p<.001$, this difference increasing over days, $F(9,324)=6.34$, $\mathrm{p}<.001$. The interaction between Phase 2 drive and Phase 3 drive was also significant, $F(1,36)=8.11$, $p<.001$. Subsequent Newman-Keuls tests $(p<.05)$ indicated that all pairs of groups differed significantly over all Phase 3 except Group HT-H-HT and Group H-HT-H.

On the last day of Phase 3, Group H-H.H was significantly faster than Group HT-HT-HT (Newman-Keuls, $\mathrm{p}<.01$ ). In Phase 4, Group H-H-H was shifted to HT, Group HT-HT-HT being maintained on $H T$, and in Phase 5, both groups were shifted to $H$. Figure 4 shows the mean speed for these two groups on the last day of Phase 3 and each day of Phases 4 and 5.

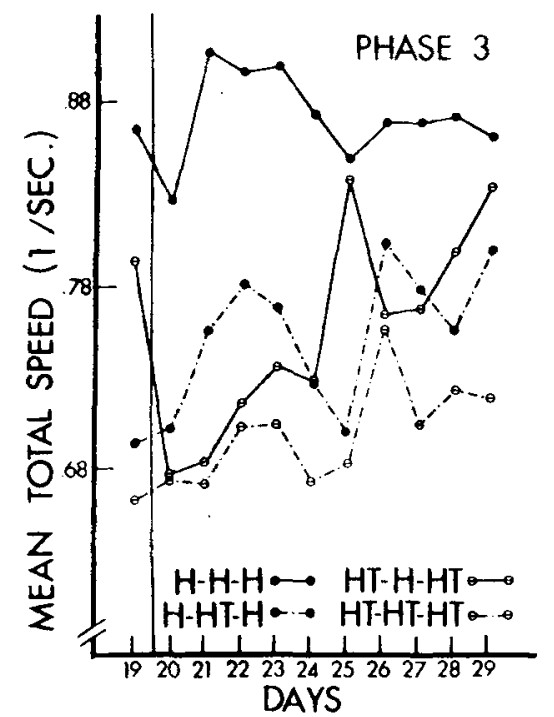

Figure 3. Mean total speed for each group on the last day of Phase 2 and each day of Phase 3.

As can be seen in Figure 4, Group H-H-H, when shifted to HT, dropped below the level of Group HT-HT-HT and remained below Group HT-HT-HT throughout all of Phase 4. Employing the last day of Phase 3 and the first day of Phase 4 as the within factor in a Days by Groups analysis of variance, the Days by Groups interaction was significant, $F(1,18)=7.68, p<.02$, indicating that Group H-H-H-HT dropped in speed from the end of Phase 3 to the first day of Phase 4 to a greater extent than did Group HT-HT-HT-HT. Over all of Phase 4, the difference between the two groups was significant, $F(1,18)=19.99, p<.001$, the size of the difference increasing with days, $F(7,126)=6.83, p<.001$. In Phase 5 , when both groups were shifted to $H$, as can be seen in Figure 4, both groups initially dropped in speed, and then Group HT-HT-HT-HT-H increased in speed at a slightly faster rate than did Group H-H-H-HT-H. Employing the last day of Phase 4 and the first day of

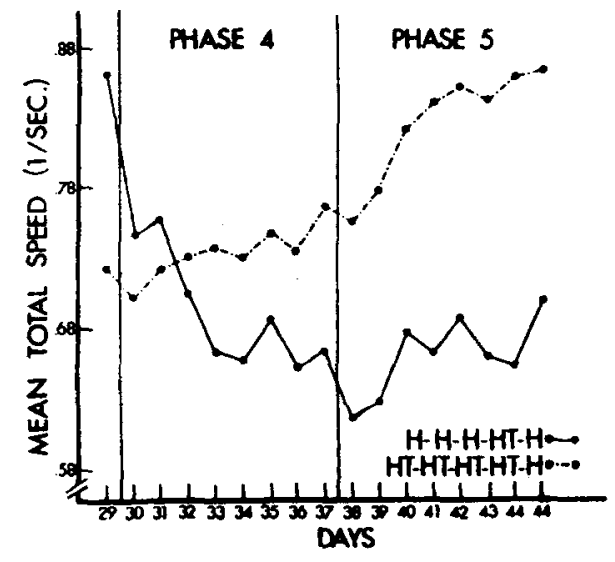

Figure 4. Mean total speed for each group on the last day of Phase 3 and each day of Phases 4 and 5. 
Phase 5 as days in a Days by Groups analysis of variance, the Days by Groups interaction was not significant, $F(1,18)=1.12, p>.30$, reflecting the fact that both groups dropped in speed initially when shifted to $H$. Over all of Phase 5 , the groups continued to differ, $\mathrm{F}(1,18)=223.63, \mathrm{p}<.0001$, both groups increasing in speed, $F(7,126)=8.32, p<.0001$, the rate of increase in speed not differing by group, $\mathrm{F}<1$.

The mean body weights of animals maintained under $\mathrm{H}$ and those maintained under HT never differed by more than $6 \mathrm{~g}$ at any time throughout the study.

\section{Discussion}

The results obtained in Experiment II with shifts from $\mathrm{H}$ to HT were highly similar to those obtained with shifts from large to small reward (e.g., Capaldi \& Lynch, 1967). Animals maintained under $H$ ran faster than animals maintained under $\mathrm{HT}$, a shift from $\mathrm{H}$ to $\mathrm{HT}$ produced a decrease in performance to a level below that of an HT control group (Phases 2 and 4), and preceding a shift from $\mathrm{H}$ to HT by training with HT eliminated the drop below the level of the HT control group when drive was shifted from H to HT (Group HT-H-HT, Phase 3). However, the results obtained in Experiment II with shifts from HT to $\mathrm{H}$ were unlike those obtained with shifts from small to large reward. Animals shifted from small to large reward increased rapdily in speed, and preceding the shift from small to large reward by training with large reward increases the rapidity of the upshift in speed (e.g., Capaldi \& Lynch, 1967; Wagner \& Thomas, 1966). However, in Experiment II, a shift from $\mathrm{HT}$ to $\mathrm{H}$ produced a continuous slow increase in speed and preceding the shift from $\mathrm{HT}$ to $\mathrm{H}$ by trials with $\mathrm{H}$ (Group H-HT-H) led to an extremely slow increase in speed. In Experiment II, a group trained H-HT-H continued to run slower than a group trained HT.HT.H throughout 24 trials (Phase 5), with no sign of this difference decreasing.

The great similarity of the results of Experiment II to those obtained with manipulations of reward magnitude can be taken to suggest that the two manipulations do influence instrumental performance through common underlying mechanisms. Simultaneously, the differences between the results obtained in Experiment II and those obtained with manipulations of reward magnitude suggest that the two variables are not functionally identical. The specific form of the results obtained in Experiment II, as compared to previous reward magnitude results, allows inferences regarding in what ways reward magnitude manipulations and the $\mathrm{H}$ vs. HT manipulation may be viewed theoretically as similar and different.

It was suggested in the discussion section of Experiment I that the within-subjects manipulation of $\mathrm{H}$ vs. HT could be viewed as a form of differential conditioning in which a large reward value is associated with the cues characteristic of $\mathrm{H}$ and a small reward value is associated with the cues characteristic of HT. Applying this analysis to the results of Experiment II supplies an explanatory basis for the similarities and differences between the results of Experiment II and those obtained when reward magnitude is varied. Consider Group H-HT-H. Following Phase 1, the stimuli characteristic of $\mathrm{H}$ would be associated with a large reward value. In Phase 2, response decrement for Group H-HT-H below the level of an HT control group would be expected on two grounds: stimulus generalization decrement due to the introduction of thirst and inhibition due to receiving a small reward value in the presence of an expectation of a large reward value (e.g., Capaldi \& Freese, 1974). Both of these factors have also been assumed to underlie the drop of a group shifted from large reward to small reward below the level of a small reward control group (e.g, Capaldi, 1974). Thus, the results obtained with shifts from $H$ to HT in Experiment II may be attributed to the same factors assumed to underlie results obtained with shifts from large to small reward. Returning to Group H-HT-H, inhibition would thus be conditioned to the stimuli characteristic of HT in Phase 2. This analysis provides an explanation of the slow upshifts obtained when Group H-HT-H was returned to $H$ in Phase 3 (and the results obtained in Phase 5). The stimuli characteristic of $\mathrm{H}$ were present in Phase 2 (in compound with the stimuli characteristic of thirst) and thus inhibition was conditioned in Phase 2 to the stimuli characteristic of $\mathrm{H}$. Accordingly, despite the increase in reward value when thirst is removed for Group H-HT.H, animals continued to run slowly because a large portion of the stimulus complex present in Phase 3 was conditioned to inhibition in Phase 2. In a reward magnitude investigation, although inhibition is conditioned to the stimuli characteristic of small reward when reward magnitude is shifted from large to small, the stimuli characteristic of small reward are not present in a subsequent test phase with large reward. Thus, more rapid upshifts would be expected when animals are trained large reward, small reward, large reward in three phases than when animals are trained H-HT-H,because of the greater similarity of the stimuli present in Phases 2 and 3 when $\mathrm{H}$ and HT are employed than when large and small rewards are employed.

Thus, the present results may be interpreted as indicating that the $\mathrm{H}$ vs. HT manipulation affects performance in the same manner as manipulations of reward magnitude, the primary difference between the two manipulations being the greater similarity between the stimuli produced by $\mathrm{H}$ and HT than between the stimuli produced by large and small rewards. Other data also suggest that the stimuli produced by $\mathrm{H}$ and $\mathrm{HT}$ are more similar than the stimuli produced by large and small reward. For example, while drive discrimination 
between hunger and thirst (presumably more similar than $\mathrm{H}$ and $\mathrm{HT}$ ) is very difficult for the rat (e.g., Amsel, 1949; Bailey, 1955; Levine, 1953), discrimination between reward related cues is relatively easy for the rat (e.g., Bloom, Williams, \& Metze, 1973; Pschirrer, 1972; Wolach, Sayeed, \& Foster, 1972). Although procedures in these studies differed, the results are consistent with the interpretation offered here.

The possibility that the HT animals were less hungry than the $\mathrm{H}$ animals cannot be ruled out. But since the same amount of food was consumed and body weight did not vary under $\mathrm{H}$ and $\mathrm{HT}$, it seems unlikely hunger varied. Even if HT animals were less hungry, an interpretation of these results in terms of reward value would be feasible because considerable evidence suggests that the reward value of food varies as a function of hunger (see Bolles, 1970). Thus, the present results may be interpreted as indicating that the reward value of food is less under HT than under $H$ whether the difference in reward value is produced by a reduction in hunger under HT or by the addition of thirst. These results supplement current views of motivation which postulate effects of relevant drives (those reduced in the situation) on reward value (e.g., Revusky \& Garcia, 1970 ) in that the present results suggest that irrelevant drives (those not reduced in the situation) also affect reward value. More importantly, the present results indicate that the effects of a strong irrelevant thirst on food-rewarded performance may be interpreted as theoretically similar to the effects of reward magnitude on instrumental performance.

A number of other questions remain to be answered. For example, the effects of weak irrelevant thirst on food-rewarded performance appear to be different from the effects of strong irrelevant thirst, weak-irrelevant thirst in some cases facilitating food-rewarded instrumental performance (e.g., Bolles \& Morlock, 1960 ), which could suggest the reward value of food is higher under $\mathrm{H}$ plus weak irrelevant thirst than under $\mathrm{H}$. And the effects of other irrelevant and relevant drives need to be evaluated. In addition, it would seem of importance to determine whether irrelevant thirst produces effects similar to those of reward magnitude through precisely the same mechanism as reward magnitude produces its effects on performance. Although the present results suggest that reward value is reduced both by reducing reward magnitude or by adding thirst to hunger, whether these two manipulations reduce reward value in the same manner is not known. For example, thirst may produce an aversive reaction to food as suggested by, for example, Levine (1956), which subtracts from the positive value of food, an effect which reward magnitude may not have. The results of the present experiments do, however, support the general approach of interpreting the effects of irrelevant drives in terms of effects on reward value.

\section{REFERENCES}

Amsel, A. Selective association and the anticipatory response mechanism as explanatory concepts in learning. Journal of Experimental Psychology, 1949, 39, 785-799.

Bailey, C. J. The effectiveness of drives as cues. Joumal of Comparative and Physiological Psychology, 1955, 48,
$183-187$.

Bloom, J. M., Williams, D. T., \& Metze, L. P. Effects of vaned and partial reward on discrete-trial patterning of rats, Animal Learning \& Behavior, 1973,1,167-170.

Bolles, R. C. Interactions with motivation. In M. H. Marx (Ed.), Learning: Interactions. London: MacMillan, 1970.

Bolles, R. C., \& Morlock, H. Some asymmetrical drive summation phenomena. Psychological Reports, 1960, 7 , 373-378.

Capaldi, E. J. Partial reinforcement either following or preceding consistent reinforcement: A case of reinforcement level. Journal of Experimental Psychology, 1974, 102, 954-962.

Capaldi, E. J., \& Freese, M. R. Partial reward training level and reward magnitude: Effects on acquisition and extinction. Learning and Motivation, 1974, 5, 299-310.

Capaldi, E. J., \& Lynch, D. Repeated shifts in reward magnitude: Evidence in favor of an associational and absolute (noncontextual) interpretation. Journal of Experimental Psychology, 1967, 75, 226-235.

Kendler, H. H. Drive interaction; 1. Leaming as a function of the simulataneous presence of the hunger and thirst drives. Journal of Experimental Psy chology, 1945, 35, 96-109.

Kendler, H. H., \& Law, F. E. An experimental test of the selective principle of drive stimuli. Journal of Experimenta? Psychology, 1950, 40, 299-304.

Levine, $S$. The role of irrelevant drive stimuli in leaming. Journal of Experimental Psychology, 1963, 45, 310-316.

Levine, $S$. The effects of a strong irrelevant drive on learning. Psy chological Reports, 1956, 2, 29-33.

Pschirrer, M. Goal events as discriminative stimuli over extended intertrial intervals. Journal of Experimental Psy chology, 1972. 96, 425-432.

Revusky, S., \& Garcia, J. Learned associations over long delays. In G. H. Bower (Ed.), The prychology of learning and motivation (Vol.4). New York: Academic Press, 1970. Pp. 1-84.

Verplanck, W. S., \& Hayes, J. R. Eating and drinking as a function of maintenance schedule. Joumal of Comparative and Physiological Psychology, 1963, 46, 327-333.

Wagner, A. R.. \& Thomas, E. Reward magnitude shifts: A say ings effec1. Psy chonomic Science, 1966, 4, 13-14.

Wolach, A. H. Sayeed, H., \& Foster, K. Pattem running to differential reinforcement. Leaming and Motivation, 1872, 3, 500-508.
(Received for publication June 24, 1974; revision received January 6,1975 .) 